\title{
Understanding Mental Workload: From a Clarifying Concept Analysis Towards an Implementable Framework
}

\author{
Bram B. Van Acker ${ }^{\text {ab* }}$, Davy D. Parmentier ${ }^{\mathrm{a}}$, Peter Vlerick ${ }^{\mathrm{b}}$, Jelle Saldien ${ }^{\mathrm{ac}}$ \\ ${ }^{a}$ Department of Industrial Systems and Product Design, Faculty of Engineering and Architecture, Ghent \\ University, Graaf Karel de Goedelaan 5, 8500, Kortrijk, Belgium \\ ${ }^{\mathrm{b}}$ Department of Personnel Management, Work and Organizational Psychology, Faculty of Psychology and \\ Educational Sciences, Ghent University, Henri Dunantlaan 2, 9000, Ghent, Belgium \\ ${ }^{c}$ Research group IMEC-MICT-Ghent University, De Krook, Miriam Makebaplein 1, 9000 Ghent, Belgium \\ * Corresponding author: bramb.vanacker@ugent.be,+32 4785714 11, orcid.org/0000-0002-6565-3569
}

Acknowledgments: This work was funded by the government agency Research Foundation Flanders (FWO) as part of the SBO project Yves of Flanders Make, Oude Diestersebaan, 133, 3920 Lommel.

Abstract: The growing need for mental workload (MWL) optimization on the shop floor yields an impressive increase in theoretical and applied references to the concept of mental workload (Young et al. 2014). However, do we really understand and agree upon what mental workload exactly is? Does it include emotional load? Can we rely upon an explanatory framework? The present account first runs a critical concept analysis on mental workload, based on the Walker and Avant (2011) method. Results show that existing definitions and theoretical accounts arbitrarily include and exclude defining variables and describe these variables on various levels of abstraction, misuse pivotal terms such as mediation and moderation, and do not theoretically explicitate the role of yet repeatedly operationalized emotional load variables such as frustration. We therefore clarify the concept by disentangling MWL into its antecedents, defining attributes and consequences. Next, we derive a clearcut conceptual definition and present a generic explanatory framework - the latter extended with insights from Cognitive Load Theory (Sweller 1988; 1994). We conclude with a set of suggestions for future research and practice. Next to contributing to the theoretical clarification of this hallmark concept, the concept analysis and derived explanatory framework, as proposed, can foster solid research practices and support practitioners in contextualizing MWL-assessment and in effectively optimizing MWL.

Keywords: mental workload, concept analysis, definition, cognitive load, framework

\section{Author final peer reviewed version}




\section{Introduction}

Throughout the history of the ergonomics and human factors field, the concept of mental workload (MWL) has been playing a substantive role. Today, MWL has even become more pivotal due to the exponential evolution of technology and the arising mass customization work context. Indeed, the introduction of top-notch technology such as augmented and virtual reality, wearable sensors and highly agile and intelligent assistive robots raises urgent questions on how to safeguard employees' mental well-being and personal efficacy, and how to transform the future operator into a flourishing knowledge worker. Optimization of MWL could therefore not be more imperative today (cf., Parasuraman and Wilson 2008). Recently, Young et al. (2014) provided an essential overview of the concept's current state-of-science. As these authors and others (cf., Xie and Salvendy 2000; Staal 2004; Matthews et al. 2015a; Mandrick et al. 2016) however point out, the understanding of mental workload still vastly lacks theoretical coherence. As Longo (2015) notes, various definitions have been suggested, but all include different workload variables based on different fields of application, beliefs, and even intuition (see also, Young and Stanton 2002). Measures derived from these definitions then do not move beyond ad hoc operationalizations, in turn reifying the theoretical conceptualization of MWL (see also the discussion on structuralism in Gerjets et al. 2009, and representationalism and operationalism in de Winter 2014). As a result, all these different, arbitrary conceptualizations only add to the confusion about the understanding of MWL (e.g., Are emotional reactions always present or not? What exactly are we now measuring?). The absence of a clear conceptual definition and an account of the processes determining and evolving from MWL hence structurally impedes solid theoretical and practical accumulation. If there is no clear theoretical understanding of MWL, practical implementations for MWL optimization will indeed repeatedly fail to be successful (Matthews et al. 2015).

Accordingly, the current literature urgently requires a critical reconsideration of the conceptualization of MWL. Here, we endeavor to contribute to this challenge. To scrutinize the current understanding of MWL and to reveal its underlying building-blocks, our account first runs a clarifying concept analysis. In a second part, we aspire to contribute to profound theory-building and measurement validation by deriving a clear-cut conceptual definition (cf., Wacker 2004). In the last part, we finalize our findings into an implementable explanatory framework of MWL, a table summarizing the current key anomalies in the literature along with their possible consequences, and some suggestions for future research and practice.

\section{Concept analysis}

Concept analysis is a rigorous process in which concepts are systematically scrutinized in order to then formulate clear-cut conceptual definitions. Such delineated definitions allow researchers to pinpoint phenomena, to map their properties and to discover and investigate relationships between them, this, to finally drive theory development forward (Walker and Avant 2011). We opt for the most widely used and well-structured Walker and Avant (2011) method unrolling eight predetermined steps. The first two steps, i.e., determining the concept of analysis and determining the aims and the purpose of the analysis, have already been covered in the introduction. The remaining steps are more elaborately touched upon and include: describing the uses of the concept, identifying the defining attributes, antecedents and consequences of the concept, identifying a model case, borderline case, related case, contrary case, (optionally) an invented case and an illegitimate case, and finally, identifying the empirical referents.

As input to the concept analysis methodology, first, a literature study is to be performed collecting all relevant sources. In our case, a literature search using the keywords 'mental workload', 'mental load' and 'cognitive load' was performed consulting the databases Web of Science (Core Collection), Google Scholar, IEEE Xplore Digital Library and APA PsychNET. Inclusion criteria were peer-reviewed journal articles, conference papers, books and technical reports, written only in English and discussing the concept directly. Exclusion criteria were book reviews and a substantial amount of empirical papers citing already retrieved theoretical references for a definition and understanding of the concept. No literature sources were excluded by time limit. We screened all articles on relevance by reading the titles and abstracts. Of the articles identified as relevant, we also hand searched the reference lists for more sources. A final sample of 82 references were included in the following concept analysis. 


\subsection{Uses of the concept}

Standard dictionaries do not account for the concept of mental workload (cf., Stevenson 2010). Indeed, the concept resides in more specialized areas and is mostly used in the context of ergonomics, human factors, educational psychology and cognitive sciences. According to The Dictionary for Human Factors/Ergonomics (Stramler 1992, p. 202), for instance, MWL is understood as "any measure of the amount of mental effort required to perform a task". Within this field, the concept is directly linked to performance criteria such as quality and human safety (cf., Young et al. 2014). Measurement validation and designing interventions to optimize this link constitutes the operational center of application in this field.

Although coined as 'cognitive load', instruction and education research addresses a very similar concept. As does the ergonomics and human factors field, research and practice in this area strives to optimize this load, but aims at fostering learning as well, next to performance. Cognitive load theory (Sweller 1988; 1994) and cognitive theory of multimedia learning (Mayer and Moreno 2003) interestingly maps effects of inefficient instruction design on performance and learning and develops strategies to overcome them. For example, the splitattention effect reveals that procedural instructions cause people to divide their attention between different types of information presented (e.g., textual information and graphical information) - increasing cognitive load. Contrary, integrating instead of separating this information does not provoke extra integration by working memory, resulting in a smaller increase in cognitive load. The modality effect then, increases cognitive capacity by consulting the visual and auditory modalities of working memory both simultaneously, alternatively to only relying on one processor. The logic for this effect is to be found in the cognitive architecture (cf., Baddeley 1992, revealing the existence of a visual-spatial sketch pad and a phonological loop, see below). Case in point is Tindall-Ford et al. (1997) finding that audio/visual electrical engineering instructions showed superiority to visual/visual instructions. A final example of such an effect, is the so-called signaling effect in which instructions are enriched by arrows, an organizing map or by stressing key words. Especially in multimedia settings, instructions enriched by such signals guiding the learner's cognitive processes, showed to result in better problem-solving performance (cf., Experiment 3 in Mautone and Mayer 2001).

Some researchers in cognitive sciences, finally, also speak of cognitive load, but frame the concept as the load being induced by a secondary task. Research on short-term memory, for instance, induces this cognitive load in order to learn more about functionalities such as memory-trace reactivation or repair (Ricker et al. 2014). Other researchers within this field see cognitive load more broadly as an aspect of task difficulty and apply it to investigate, for example, the neural functioning of distraction and concentration (Sörqvist et al. 2016).

\subsection{Defining attributes}

Arriving back at the ergonomics and human factors field now, the defining attributes of a concept are those characteristics that appear over and over again when reviewing definitions and descriptions, and that have to be present for the concept to occur (Walker and Avant 2011). A fragment of the definitions encountered in our literature review illustrates the attributes stepping forward. First, Moray (1979) defines mental workload as "... an inferred construct that mediates between task difficulty, operator skill, and observed performance" (p. 443). More recent accounts see the concept as "How hard the brain is working to meet task demands" (Ayaz et al. 2012, p. 36) or as Young and Stanton (2001, p. 507) put it: "The level of attentional resources required to meet both objective and subjective performance criteria, which may be mediated by task demands, external support, and past experience". Such accounts thus point at the interaction of the neural capacities of the operator and the demands of a task (see also Pickup et al. 2005, for a brief overview of these two defining elements).

Other definitions take an important step further in specifying this interaction. Andre (2001, p. 377), for example, thinks of MWL as, ".... a hypothetical construct that represents the cost incurred by a human operator to achieve a particular level of performance". Also Gopher and Donchin (1986, as quoted by Staal 2004, p. 15) consider this costly aspect when defining 'workload' as "...intended to capture limitations on the operator's information processing apparatus ...", as did Kramer et al. (1987, p. 146) speaking of “... the cost of performing one task in terms of a reduction in the capacity to perform additional tasks, given that the two tasks overlap in their 
resource demands". Here, the notion of cost and limitation is added to the understanding of the cognitive capacities involved and helps delineating a first attribute defining mental workload as:

\section{Spending Cognitive Resources}

Employees' cognitive resources are physiological in nature, have, as predisposed biologically, a finite capacity and in essence consist of multiple independent resources. When these resources are being spend, they entail the attention and effort delivered towards work related stimuli or information one is exposed to at work. Multiple Resource Theory (Wickens 2002, 2008) states that the allocation of cognitive resources varies during the course of information processing. Specifically, this theory predicts that MWL will be lower when task demands tap into less common components of innate resources (see below). When for instance simultaneously presenting auditory instructions while performing a visual detection task, more information can be processed without impeding performance (Wickens 2002, 2008). The stimuli being processed in working memory (as determined by the work demands, see below) is what we speak of when addressing this first defining attribute.

The following working memory functions for allocation and monitoring then allow for the processing of these stimuli. First, attentional processes deploy different modes (Wickens and Hollands 2000; Wickens and Carswell 2012): selective attention concerns what stimuli to process, focused attention covers sustained processing and avoiding distraction, while divided attention entails processing multiple stimuli at once. Vigilance, interestingly, is a specific kind of prolonged attention (Warm et al. 2008; Langner and Eickhoff 2013). Secondly, in order to subsequently process (e.g., detect) stimuli, perception and memory resources are relied upon, while motor control resources support action execution (Stork and Schubö 2010; Proctor and Vu 2012; Wickens and Carswell 2012; for an interesting predictive processing account in driving, see Engström et al. 2017). Finally, executive control (as referred to by Salvendy 2012) coordinates all these working memory processes and specifically encompasses strategic management functions such as reorganization, chunking, inhibition of irrelevant information, allocation of (more/less) resources, decision making and action planning (cf., Proctor and Vu 2012).

MWL is hence essentially a constantly varying measurable physiological state directly related to working memory processes monitoring and allocating resources (cf., Xie and Salvendy 2000; Mandrick et al. 2016; see also the malleable attentional resources theory, Young and Stanton 2002). Operators will then typically show to function at optimal levels of MWL (cf., Hancock and Warm 1989) or suboptimal levels (underload and overload), eventually affecting their performance and other outcomes (Hart and Wickens 2010; Wickens and Tsang 2014; see also the performance 'redlines' of mental workload in Young et al. 2014).

Altogether, these cognitive processes are considered to lie on a continuum between automatic processing (i.e., fast, unconscious and almost without attention) and controlled processing (i.e., high attention and high effort; Schneider and Shiffrin 1977). Personal and contextual differences (i.e., moderators, see below) such as experience and assistive technology, or antecedents (also see below) such as task difficulty influence where the resources being spend then actually reside on this continuum, and, how the expenditure of these resources is being experienced by an employee.

Zooming out again, Staal (2004) correctly notices that definitions as presented above show similarities with early accounts of stress (i.e., the stimulus-based approach; cf., Young et al. 2014, for a similar perspective), but notes that they do not take into account a subjective experience. Hilburn and Jorna (2001) already tried to bridge this gap by making a distinction between task load and workload, in which the former entails the load imposed by the task and the latter comprises the subjective experience. Other accounts speak of a feeling of being loaded (Johannsen 1979) or the perception of load (Pickup et al. 2005). Although not abundantly present in existing definitions, we choose to take up this subjective experience as a second defining attribute because of its recurrent operationalization in the most widely used subjective questionnaires such as Hart and Staveland's (1988) NASATLX (see de Winter 2014, for search results on the most popular MWL questionnaires and a critique on the NASATLX). We hereby delineate the second attribute emerging from our literature review, defining mental workload as: 


\section{Triggering a Subjective Experience}

Despite of its widespread presence in MWL assessment, the literature is very ambiguous as to how it relates to this subjective experience. The sparsely available conceptions do not only point at the direction of a feeling or perception of being loaded (Johannsen 1979; Pickup et al. 2005), but also at a related situation appraisal (McGrath 1976; Fairclough 2001; see, appendix A in Staal 2004). The latter is thought of as a continuous reappraisal process of the work demands and is suggested to regulate the mental effort invested (Hockey 1997; Fairclough 2001; Matthews et al. 2015). This subcortical pre-conscious process does so by probing the current performance level, arousal and affective responses (e.g., frustration, comfort) to then allocate attentional and other cognitive processing resources (Crawford and Cacioppo 2002; Matthews et al. 2002; Young and Stanton 2002; Langner and Eickhoff 2013). The MWL experienced hence affects the resources being invested (Hancock and Warm 1989; Zijlstra 1993; Fairclough 2001). It still remains unclear however how the ergonomics and human factors field then fundamentally delineates this experience and, importantly, whether emotions are part of MWL or not. The latter is crucial, because, intriguingly, on an operational level we do see a widespread presence of emotional load when addressing MWL. Case in point is that the bulk of the most highly cited empirical publications on MWL relies on the NASA-TLX (Hart and Staveland 1988; de Winter 2014; Estes 2015) of which one of six items measures frustration and notions of stress and insecurity. Thus, although definitions of MWL do not include emotions, emotions are being operationalized.

Here, we propose to introduce a distinction between MWL and emotional load (EL) and to not include EL in the definition of MWL. We do so, because EL is not always present when exposed to work demands (McGrath 1976; Gaillard 1993; Staal 2004). EL in fact exists as a function of, i.a., a situation appraisal on coping ability (cf., McGrath 1976; Matthews et al. 2002). More precisely, the occurrence of EL depends on the interaction between the work demands, the perceived ability to cope with those demands, and the importance of being able to cope. Indeed, if one thinks (i.e., foresees) he or she can cope with the work demands or does not find the ability to cope important, emotions will not necessarily be present (i.e., not exceeding neutral values on a subjective rating scale, for instance). Emotional processing and a subjective emotional experience is hence no conditio sine qua non in neural processing attending to work demands (see also Staal 2004, referring to primary and secondary workload factors). As a consequence, emotional variables such as frustration, discomfort or excitement should be no part of any defining attribute nor definition of MWL. The second defining attribute is thus to be understood as a mere cognitive awareness of the physiologically embedded cognitive resources being spend. We will nevertheless show that emotional load still plays an important role in the detection of suboptimal MWL (i.e., underload or overload) and that it can indirectly affect MWL-levels and coping behavior.

To conclude the current part covering the defining attributes, it is evident that both defining attributes will not occur without the presence of work demands however, as repeatedly portrayed in the array of definitions presented above. Because of its prevalence in the literature, the latter could be included as one of the attributes as well, but according to the methodology of Walker and Avant (2011), an attribute cannot be an antecedent at the same time, nor a consequence. The role of work demands will therefore be classified under antecedents.

\subsection{Antecedents}

\section{Cognitive work demands}

Antecedents are those components or situations preceding a concept and making its existence possible (Walker and Avant 2011). Cognitive characteristics or demands of one's work constitute a first emerging antecedent of MWL. A first example is task complexity (e.g., Moray 1979; Wickens 2002). Low task complexity can yield more automatic processing, hence lowering MWL. In manual assembly, for instance, time to completion empirically relates to the physical properties of objects such as number of symmetrical planes or redundant holes (Richardson et al. 2004; Richardson et al. 2006; see also Layer et al. 2009 for a coding taxonomy in operator decision making). Incidental variables such as system failures, distractions or environmental changes (e.g., noise, temperature) can be seen as another part of task complexity (Hart and Staveland 1988; Pickup et al. 2005; Shaw et al. 2018). Task switching is a second exemplary case of cognitive work demands. When (rapidly) switching to 
new (sub-)tasks, different resources have to be relied upon and several mental models have to be kept in mind simultaneously (Neerincx et al. 2000; Proctor and Vu 2012; Wickens 2017). Finally, cognitive work demands such as the perceptual format of the instructions (e.g., visual/auditory, digital/paper) might affect MWL levels as well (cf., Wickens 2002, 2008). In all, cognitive work demands represent all objective stimuli in the work environment that elicit the spending of cognitive resources in employees. These stimuli can hence be managed, changed and objectively measured. As self-induced cognitive demands such as employees' curiosity or high learning motivation are not fully under control of an employer, such person-related cognitive demands do not belong to cognitive work demands. Also, primary physical or emotional stimuli and demands at work are not cognitive work demands as they mainly elicit, respectively, physical effort (e.g., lifting a heavy weight) and/or emotional effort (e.g., providing emotional social support, crying, etc.).

\section{Cognitive architecture}

The predisposed human cognitive architecture comprises a second antecedent. Within the ergonomics field, Multiple Resource Theory (MRT) (Wickens 2002, 2008) is the most dominant theory relied upon. Wickens' MRT proposes that human beings have, instead of just one common pool, several differentiated, but limited pools of cognitive resources that can be tapped into simultaneously. These multiple resources are used for various mental operations, ranging from sensory-level processing to meaning-level processing (Basil 2012) and, more specifically, are allocated across different tasks touching upon different sensory modalities (e.g., auditory vs. visual), types of memory code (visual vs. spatial), stages of information processing (e.g., perceptual/cognitive vs. response) and channels of visual information (e.g., focal vs. ambient).

When simultaneously addressing similar resources, MRT shows, certain tasks will suffer from suboptimal cognitive processing, leading to poor performance. When tapping into different resources at the same time, to the contrary, performance levels can end up higher (Wickens 2002, 2008). The way work demands tap into this human cognitive architecture will thus determine the amount of cognitive resources spent and how an employee will experience this mental workload. Dominant theories in the ergonomics literature and the human factors field frame this interaction in terms of demand/resource balances or stress/strain dynamics (cf., Young et al. 2014).

Summarized, the various cognitive resources available to human beings are limited on themselves, while the total amount of cognitive resources spent can be extended based on their multidimensionality. In turn, however, these multiple cognitive resources are considered to draw from the same underlying pool of energy resources as do emotional resources and physical resources (Kahneman 1973; Norman and Bobrow 1975; Mandler 1979). This way (see some cases below), these three load factors can in fact draw resources away from each other.

\subsection{Consequences}

The literature on MWL is mostly centered around its optimization, since overload and underload can lead to, e.g., frustration and fatigue (Matthews et al. 2013; Matthews 2016), safety risks, errors, quality loss and time loss (cf., Hart and Staveland 1988; Brookhuis and de Waard 2000; Young et al. 2014; Wickens 2017), and lowered performance (Hancock 1996). In the long run, optimizing these levels ultimately affects employees' job satisfaction and physical and mental health outcomes such as psychological well-being, burnout and absenteeism (cf., Young et al. 2014).

We refer the reader to Table 1 for an overview of key references in the literature providing the insights for the three core levels of analysis of the MWL concept we have covered up to this point. Next, we will proceed with some concrete cases helping to delineate MWL and with a short overview of how MWL is being assessed empirically. 
Table 1 Current MWL literature: Overview of key references for the three core levels of analysis of the MWL concept

\begin{tabular}{|c|c|}
\hline $\begin{array}{l}\text { Core Level of } \\
\text { Analysis }\end{array}$ & Key references \\
\hline Defining Attributes & $\begin{array}{l}\text { Andre (2001), Ayaz et al. (2012), Crawford and Cacioppo (2002), de Winter (2014), Engström } \\
\text { et al. (2017), Estes (2015), Fairclough (2001), Gaillard (1993), Gopher and Donchin (1986), } \\
\text { Hancock and Warm (1989), Hart and Staveland (1988), Hart and Wickens (2010), Hilburn and } \\
\text { Jorna (2001), Hockey (1997), Johannsen (1979), Kramer et al. (1987), Langner and Eickhoff } \\
\text { (2013), Mandrick et al. (2016), Matthews et al. (2002, 2015), McGrath (1976), Moray (1979), } \\
\text { Pickup et al. (2005), Proctor and Vu (2012), Salvendy (2012), Schneider and Shiffrin (1977), } \\
\text { Staal (2004), Stork and Schubö (2010), Warm et al. (2008), Wickens (2002, 2008), Wickens and } \\
\text { Carswell (2012), Wickens and Hollands (2000), Wickens and Tsang (2014), Xie and Salvendy } \\
\text { (2000), Young and Stanton (2001), Young et al. (2014), Zijlstra (1993) }\end{array}$ \\
\hline Antecedents & $\begin{array}{l}\text { Basil (2012), Hart and Staveland (1988), Kahneman (1973), Layer et al. (2009), Mandler (1979), } \\
\text { Moray (1979), Neerincx et al. (2000), Norman and Bobrow (1975), Pickup et al. (2005), Proctor } \\
\text { and Vu (2012), Richardson et al. (2004), Richardson et al. (2006), Shaw et al. (2018), Wickens } \\
(2002,2008) \text {, Wickens (2017), Young et al. (2014) }\end{array}$ \\
\hline Consequences & $\begin{array}{l}\text { Brookhuis and de Waard (2000), Hancock (1996), Hart and Staveland (1988), Matthews (2016), } \\
\text { Matthews et al. (2013), Wickens (2017), Young et al. (2014) }\end{array}$ \\
\hline
\end{tabular}

\subsection{Model case}

A model case presents a paradigmatic, pure and real-life example, and entails all of the defining attributes (Walker and Avant 2011). To structure our model case - here applied to manual assembly - we borrow from Stork and Schubö (2010) covering the entire process of MWL starting from perception, attention and memory, and taking it to action planning and execution. In support of ecological validity, we also follow the example of Galvin et al. (2017) by including at least one antecedent (first paragraph) and one consequence (third paragraph).

Yves is a 56-year old worker at a manufacturing plant. Today he has to perform a complex assembly he has never encountered before. The instructions are projected onto his work station in augmented reality.

He looks at the parts and the instructions and starts to figure out how the parts should be assembled. The first stage in doing so entails perception. The properties of the parts are being processed (stimulus preprocessing) and Yves locates (feature extraction) and identifies (stimulus identification) all parts with relevant properties. Subsequently, he identifies all relevant fastening points. Yves is now set to make a decision (executive control) on where to start joining the parts. Every now and then he is aware that he is thinking hard. But he manages and continues, and eventually arrives at the stages of response selection and action. The action tendency to now execute the first joining action is organized by the cognitive neural programming for motor actions. Concretely, a grasping movement and an orienting movement is planned. Cognitive resources manage the muscular processes to finally complete the correct joining movement (action execution) and adjust for pressure and orientation while doing so (motor adjustment).

\section{The result is a first perfectly joined assembly step without making any errors in the course of it.}

This model case reveals that MWL spans from stimulus perception and processing, towards the decisionmaking process of response selection and finally motor execution and adjustment. All these stages are propelled by allocation and monitoring processes as unfolded above.

\subsection{Related case}

The model case could have evolved differently, however. Let us imagine that the complexity of the assembly was higher because of very similar, confusing parts. More precisely, the assembly parts in this case were designed in such a (bad) way that the dimensions and properties hardly differed, making Yves ponder why the parts or the instructions could not have been designed more efficiently. Yves is motivated to do well and keeps on cognitively attending to the parts, but realizes that he cannot figure out how to join them. He gets frustrated. As a result of this negative emotional experience, Yves does not have enough cognitive resources left to try harder and 
find out how to match them (Gaillard 1993; Staal 2004). Negative emotional load (and broader, stress, for an energetic account, see Gaillard 1993) instead of mental workload has now taken over.

In this related case, the complexity of the task is suboptimal, i.e., too high for Yves, and induces a cognitive overload. The latter leads Yves to feel that he has a lack of internal control to cope with the cognitive work demands (through a situation appraisal, McGrath 1976), which then triggers frustration. We are therefore not speaking of mental workload anymore, but of emotional load. Interestingly, since both emotional and cognitive resources are thought to draw from the same general underlying pool of physiological energy resources, emotional load in this case takes the upper hand and obstructs cognitive resources to fully exploit their potential. Also cognitive work demands other than task complexity, such as instruction format, could trigger frustration. Other potential emotions that can arise when exposed to cognitive work demands are, i.a., anxiety or joy. Although these emotional experiences might be felt deeply and might influence one's work behavior, they do not belong to cognitive work demands and do not represent MWL, but emotional load instead.

In all, emotional load is a related case, because it appears very similar and contains some notions of the defining attributes, but differs when studied from up closely. Related cases help to discover how the concept relates to a closely surrounding network of concepts and help to clarify what should be included as a defining attribute and what not (Walker and Avant 2011).

\subsection{Borderline case}

Borderline cases hold most, or even all, defining attributes, but differ strongly in one of them, for instance in terms of length of time or the intensity of occurrence (Walker and Avant 2011). In most instances, cognitive effort expended and the resonating cognitive effort experienced raises as a function of the cognitive work demands. Sometimes, however, Yves performs a task that he enjoys so much, absorbing him into the task, that his actions and attention seem to flow effortlessly (Bruya 2010). Even in case of an increase in cognitive work demands (evidently up to a certain threshold), such flow state yields the same level of efficacy while experiencing no subjective self-consciousness about the effort being spend - an effect found in domains such as factory line working, chess and rock climbing (Csikszentmihalyi 1975). The defining attribute representing the cognitive subjective experience in this case hence does not raise as a function of the cognitive resources being spend. The case of the human flow state or absorption at work is consequently not the exact same concept as MWL.

\subsection{Contrary case}

A contrary case illustrates the differences with the concept being analyzed and again helps to exclude variables from the defining attributes. According to The Dictionary for Human Factors/Ergonomics (Stramler 1992, p. 202), physical load is the opposite of MWL. Since cognitive resources are actually physiological in essence, we present the following example to draw a line in-between. When Yves faces a task that he has done a thousand times before, the cognitive programming to motorically execute this task is so automated that his MWL level is optimal. The strain on his muscles and cardiovascular system, however, is what we consider physical load and this load can become suboptimal when the repeated movements cause strain or when, for instance, the physical weight of the task components is too high.

As with the related case example, interestingly, also physical load can interfere with mental workload. Since also physical load draws from the same underlying energy pool, participants in various experiments estimated the duration of the experiment (i.e., a task consuming cognitive resources) as shorter when they were, e.g., exerting physical effort on a dynamometer (for a meta-analysis, see Block et al. 2016).

\subsection{Empirical referents}

Empirical referents are to be related directly to the defining attributes and represent categories of phenomena or measures that reveal the occurrence of the concept (Walker and Avant 2011). Resonating with the multidimensional nature of MWL, its measures are equally various in nature. Some authors divide them into four categories (cf., Gopher and Donchin 1986, others do so in empirical and analytical methods, see Xie and Salvendy 2000). In general, the literature converges towards assessing MWL with performance measures, subjective measures and physiological measures (cf., O’Donnell and Eggemeier 1986; Paas et al. 2003; Cain 2007; Wickens 
and Tsang 2014). The latter two aim directly at the defining attributes, the former indirectly aims at measuring the cognitive resources spent.

A first widely used type of gauge entails registering the subject's performance. Also called objective measures, these techniques use dependent variables such as reaction time, quality, accuracy and error rate (Paas et al. 2003) or performance on subsequent or even simultaneous secondary tasks (cf., dual-task paradigms) to make inferences about the cognitive resources spent (cf., Brookhuis and de Waard 2000; Brunken et al. 2003). Using rating scales, another range of measures gauges the subjective experience of the cognitive resources that have been spent. The NASA-TLX (Hart and Staveland 1988) and the Subjective Workload Assessment Technique (SWAT; Reid and Nygren 1988), for example, are two of the most popular scales (Vidulich and Tsang 1986; Rubio et al. 2004). Finally, a wide variety of measures starts from the knowledge that the mental processes entailing MWL are physiological in essence. In (near) real-time, electroencephalography (EEG), event-related potentials (ERP) derived from EEG and functional Near Infrared Spectroscopy (fNIRS), for instance, can provide estimations of the electrical activity or cerebral blood flow to derive MWL levels from (Antonenko et al. 2010; Ayaz et al. 2012; Mehta and Parasuraman 2013). Other measures such as electro-dermal activity (also coined 'galvanic skin response'; Boucsein 2012), heart rate variability (Aasman et al. 1987; Shakouri et al. 2018) or pupillometry (Backs et al. 2003), try to capture reactions of the autonomous nervous system to approximate MWL.

\section{Conceptual definition}

As Matthews et al. (2015) indicate, conceptualizing mental workload in terms of a general workload factor might not at all be adequate to fully grasp all MWL's nuances (see also Gopher and Donchin 1986; Pickup et al. 2005; de Waard and Lewis-Evans 2014). Indeed, there is no single 'gold standard' pinpointing a unitary latent factor of MWL, instead, mental workload is multidimensional (see also, Chen et al. 2016). A conceptual definition of MWL should therefore integrally encompass the most elementary dimensions of MWL. Derived from the concept analysis presented above, we therefore scaffold our conceptual definition onto the following four proposed elementary dimensions of MWL: cognitive work demands interacting with the human cognitive architecture, inducing cognitive physiological processing and a cognitive subjective experience. Centralizing the defining attributes and contextualizing them with the antecedents, this then rolls out into the following conceptual definition:

"Mental workload is a subjectively experienced physiological processing state, revealing the interplay between one's limited and multidimensional cognitive resources and the cognitive work demands being exposed to."

In order to fully operationalize this definition in a specific context, the task designer or ergonomist can implement this definition by addressing the exact antecedents, defining attributes, moderators and the expected work behavior and consequences as presented in Figure 1. To allow for such contextualized operationalizations, we tried to overcome the two major caveats we found in existing definitions (see section 2.2 Defining attributes for some examples). First, MWL definitions arbitrarily include and exclude key components (e.g., limitations, experience, performance). Here, we scaffolded our definition onto the four most elementary dimensions extracted from the literature. Secondly, different definitions formulate MWL on different levels of abstraction (e.g., "how hard the brain is working" and "processing status" vs. "levels of attentional resources", or "task demands" vs. "task difficulty", and "performance" vs. "subjective and objective performance"). Here, we formulated the definition on a high level of abstraction, since we showed that MWL does not only imply attentional resources, for instance. At the same time we narrowed down its scope to the limited and multidimensional cognitive resources tackling cognitive work demands. Operational definitions can thus be derived from these abstract key components, but within elementary boundaries based on the concept analysis and explanatory framework presented below. As we will show when presenting some suggestions for future work, researchers and practitioners can operationalize this definition for various contexts, but should explicitate all variables, manipulations, interactions and measures thoroughly. 
In all, this definition and its underlying concept analysis could be a first step in clarifying and strictly delineating the concept at hand while providing a common frame of reference to which different operationalizations can be systematized and compared to each other. We will now organize the findings from the concept analysis into an explanatory framework and we will take off with a final point of confusion.

\section{Explanatory framework}

When reviewing various MWL definitions, it also appears that the exact explanatory role of certain hallmark variables in the relationship between the antecedents, defining attributes and outcomes of MWL are left to the reader's interpretation. Not only does the literature often remains fuzzy on these variables (see above), if a reference is made to the role of what the psychological tradition calls moderators and mediators, these both terms are in fact often misused. Case in point is Young and Stanton (2001, p. 507) defining MWL as "the level of attentional resources required to meet both objective and subjective performance criteria, which may be mediated by task demands, external support, and past experience". Mediator variables are, according to the psychological literature, primarily internal to the human, change along with variations in the predictor variable and explain the mechanism between predictor (e.g., task complexity) and outcome (e.g., quality). Moderators (e.g., age, experience) tell when, for whom and how strong a relation deploys (Baron and Kenny 1986). In the above definition, the variable 'task demands' cannot mediate, since it is a predictor variable, i.e., an antecedent of MWL. Also the 'objective and subjective performance criteria' mentioned by Young and Stanton (2001) in their definition, are, to our understanding, task demands. 'Past experience' and 'external support' then, are actually moderators in the relationship between these predictor variables and the level of attentional resources required. These latter are mediators in the relation between the task demands and performance. To give another example, Parasuraman and Hancock (2001, p. 306) suggest that "... workload is also mediated by the individual response of human operators to the load and their skill levels, task management strategies, and other personal characteristics". Here, 'skill levels', 'task management strategies' and 'personal characteristics' are moderators, of which 'task management strategies' could also be mediators changing along the course of task execution. The mentioned 'individual response' is too broad to bring down to one category or the other, but could be a mediator, as could workload be one. Altogether, these definitions thus structurally disregard the opportunity to provide conceptual clarity, which is intelligible when no conceptual agreement nor explanatory framework on the understanding of the concept is at hand. These kind of pivotal, but unspecified variables are therefore explicitly introduced in the framework we propose (see Figure 1), as moderator and mediator variables.

To conclude, the core of the framework presented in Figure 1 is based on our concept analysis and encompasses the Antecedents, Defining Attributes and Consequences of MWL. We then added Moderator variables and Employee Work Behavior variables derived from the literature review, in order to more completely explain the process from Antecedents all the way up to the Consequences. Within these clusters of variables, we also added variables retrieved from cognitive load theory (Sweller 1988; 1994) and the technology literacy literature (cf., Venkatesh et al. 2003). When reading this framework, the following explicitations should be taken into account.

1. Spending Cognitive Resources and the elicited Subjective Experience are mediating variables, because these processes explain the relation between the Antecedents and the Employee Work Behavior. Spending Cognitive Resources and the Subjective Experience triggered constitute the core of the definition of mental workload and are what we refer to when speaking of mental workload. MWL therefore solely implies the cognitive resources that directly attend to cognitive work demands (i.e., primary workload factors in McGrath 1976 and Staal 2004) and the cognitive experience directly triggered by those work demands.

2. Two Antecedents are identified (Cognitive Work Demands and the Human Cognitive Architecture). Through their interaction, MWL will be triggered and moderating (work and employee related) variables might influence this relation. Yves' excellent visual-spatial skills, for example, will help to orient and rotate towards finding matching component pairs. Compared to other employees, Yves will consequently spend less cognitive resources and he will perceive the thinking that he is doing as fairly low. Other 
employee related moderating variables include, e.g., differences in working memory capacity (Jipp and Ackerman 2016), dexterity (Mayer and Sims 1994; Pillay 1997), technological literacy (cf., Venkatesh et al. 2003), experience, motivation and personality differences in, e.g., conscientiousness (Guastello et al. 2014), tendency to worry (Grassmann et al. 2017) and coping style (Hart and Staveland 1988; Young et al. 2014). Also, Yves could have had the possibility to, on his own initiative, consult assistive technology indicating matching assembly parts as well. Work related moderators can thus help tackling Cognitive Work Demands and also include, for example, social support, leadership style and provided job autonomy (cf., Van de Ven et al. 2008).

3. When employees (subconsciously) weigh the available Moderators against the presented Cognitive Work Demands, indirect effects (i.e., secondary workload factors in McGrath 1976 and Staal 2004) such as emotions can arise. Spending Cognitive Resources already starts from the moment the cognitive work demands are presented, the employee merely familiarizes with these demands and mentally simulates certain manipulations. Throughout this allocation and monitoring process, employees might appraise the situation as positive (e.g., "I experience that I have to think hard, but I foresee to be able to cope with the task demands."). If also the importance of being able to cope is appraised as positive (see above), positive (or neutral) emotions could emerge. If the situation is, contrarily, being appraised as threatening (i.e., low coping ability), negative emotions such as frustration or anxiety could be triggered. Emotions then affect MWL levels by drawing away resources from the underlying common pool of resources. As such, emotional load is extremely relevant in, for example, pinpointing overload and explaining performance, mental well-being and 'offloading' behavior (Staal 2004).

4. Employee Work Behavior represents the actions that are the actual physical implementations of the allocation and monitoring processes. These variables include, i.a., motor execution, the sequence of execution, accuracy, speed and safety behavior. Interestingly, when levels of processing are suboptimal (i.e., too high or too low), employees could then 'offload' through task shedding, relying on assistive technology, consulting colleagues (cf., distributed cognition, Choi et al. 2014), or finally, being apathetic or by dropping out (Matthews et al. 2002). All these actions are recurrently updated in an iterative process based on fluctuating levels of allocation and monitoring. Awareness of sub-performance at the center of this iterative process then serves as feedback information (Hancock and Warm 1989; Matthews and Campbell 2010). MWL thus arises from the moment cognitive work demands are being perceived and lasts throughout employee work behavior (including probing sub-performance), right up until task completion.

5. Finally, we added variables extracted from cognitive load theory (CLT; Sweller 1988; 1994). Based on this theory we added 'learning' to Employee Related Consequences and to the Human Cognitive Architecture part of the Antecedents and added Baddeley's (1992, 2000) multi-component model of a limited working memory containing a visual-spatial sketch pad, a phonological loop and an episodic buffer. The first of these components stores visual and spatial information, the second stores phonological information by means of maintaining a rehearsal loop (cf., the inner voice) and the latter holds information to integrate information from the first two (and possibly other information) while interacting with longterm memory. We think these two extensions are crucial since multiple resources theory, although covering the first two components, is restricted to overload situations imposed by multi-tasking (Wickens 2008). CLT however, also accounts for schema acquisition in long-term memory. These schemas interact with working memory processes through the episodic buffer. The CLT perspective thereby moves beyond ergonomics' main focus on quality, time efficiency, error, etc., and addresses how work and instruction design can not only enhance performance, but also the efficient long-term acquisition of expertise. We believe this notion is fundamental in conceptualizing MWL today and we see the ergonomics and human factors field briefly covering this CLT perspective (cf., Oviatt 2006; Richardson et al. 2006; Wickens 2017). Indeed, since top-notch technology and mass-customization requires ever more cognitive processing and learning from operators, the scrutiny on the effects extracted from cognitive load theory (see 2.1 Uses of the Concept above) can provide a strong added value to the standards of practitioners in 
the field and of researchers. Altogether, this framework presented in Figure 1 can help explain how an operator iteratively attends to a task and how this dynamically affects mental workload. 


\section{ANTECEDENTS}

\begin{tabular}{|l|}
\hline \multicolumn{1}{|c|}{ Cognitive Work Demands } \\
- task complexity \\
- task switching \\
- instruction format
\end{tabular}

Human Cognitive Architecture
- limited resource dimensions
- limited working memory components
- limited common underlying pool
of resources

\section{DEFINING ATTRIBUTES}

MWI

\begin{tabular}{|c|c|}
\hline \multicolumn{2}{|c|}{$\begin{array}{l}\text { Spending Cognitive Resources } \\
\text { • allocation and monitoring } \\
\text { Triggering a Subjective Experience } \\
\text { - awareness of resources being spent }\end{array}$} \\
\hline$\longrightarrow$ Emotion & $\mathrm{ns} \stackrel{\boldsymbol{\Lambda}}{\boldsymbol{1}}$ \\
\hline \multicolumn{2}{|c|}{ Moderators } \\
\hline $\begin{array}{l}\text { Employee Related } \\
\text { - visual-spatial intelligence } \\
\text { - experience } \\
\text { - technology literacy } \\
\text { - personality }\end{array}$ & $\begin{array}{l}\text { Work Related } \\
\text { - assistive technology } \\
\text { - social support } \\
\text { - leadership style } \\
\text { - job autonomy }\end{array}$ \\
\hline
\end{tabular}

\section{CONSEQUENCES}

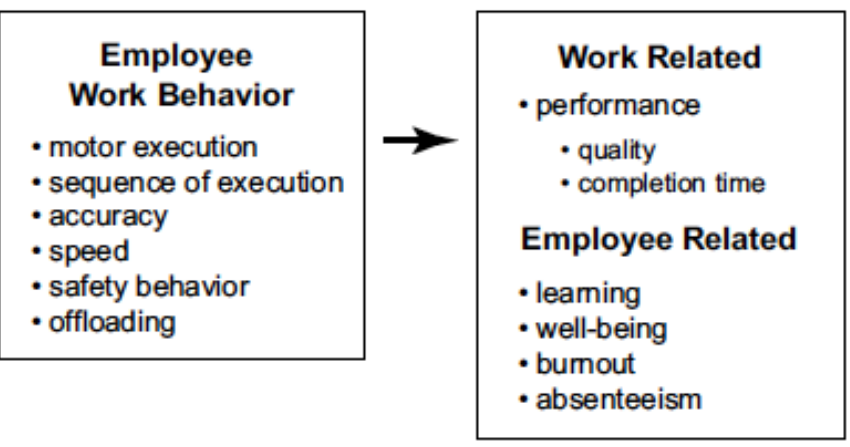




\section{Discussion}

In many fields, as in the ergonomics and human factors field, measurement of concepts seems to prevail over first designing clear conceptual definitions to build on (Wacker 2004). The latter approach serving as a strong basis for statistical analyses is however the generally agreed upon standard in order to yield high consistency between measures and their empirical data, and to guarantee the validity of measures (cf., Wacker 2004; Bollen 2010). Today, the conceptualization and operationalization of MWL differs per research account, this, caused by three anomalies that our concept analysis revealed: First (A), the literature suffers from arbitrary selections of various defining variables and a description of these variables on different levels of abstraction. Second (B), mediator and moderator variables, if considered, are often misused conceptually and not implied into an explanatory model. Third (C), emotional load variables are often (but not always) operationalized, without being addressed theoretically. In Table 2 we present an overview of these key anomalies and elaborate on possible adverse implications for research and practice.

Table 2 Current MWL literature: Overview of key anomalies and their consequences for research and practice

A. In absence of a generic theoretical framework covering the entire MWL process, the current state of science arbitrarily includes and excludes various defining variables and describes these variables on different levels of abstraction.

\section{$\rightarrow$ Consequences:}

1. Comparability and Generalizability. Confusion about what is to be included in measurement procedures leads to different operationalizations of the concept (e.g., EEG or fNIRS) rooted in different antecedents (e.g., task complexity or instruction format) yielding differential aspects of MWL (e.g., vigilance or divided attention). As a result, they cannot be compared to each other because of not being imbedded in a common framework, nor can findings be generalized to real life situations without knowing which variables might be at play there. What is often left are stand-alone post-hoc reifications of the MWL measured.

2. Addressing confounding variance. No clarity on the overall possible MWL process makes that measurement validation will always fall short of addressing possible contaminating variance.

3. Efficiency in theorization. High levels of abstraction leave room for loosely operationalizing variables, misinterpretation of data and drawing incongruent conclusions for follow-up studies.

B. Mediator and moderator variables, when investigated, are often conceptualized incorrectly and not implied into an overarching explanatory model.

$\rightarrow$ Consequences:

1. Measurement diagnosticity (cf., Matthews et al. 2015b) and predictability. There is no possibility to rely on an overview of how specific explanatory (defining) variables can help in validating measurement sensitivity (e.g., certain measures possibly being more sensitive to visual attention) and how to systematize prediction (e.g., measuring lower speed or pupil dilation due to high MWL could help in predicting errors or safety risks).

2. Accounting for boundary conditions. Measured MWL-levels within and across individuals can pan out in all directions, without researchers and practitioners knowing how certain boundary conditions (e.g., visual spatial intelligence) are altering the data obtained and how they can be (statistically) controlled for (cf., Matthews et al. 2015a) in order to find robust differences between experimental conditions, or how to cultivate them to eventually optimize MWL.

C. Emotional load is not being included in theory, though often operationalized.

$\rightarrow$ Consequences:

1. Measurement selectivity/validity ( $c f$., Matthews et al. 2015b). While emotions and cognitions draw from common resources, the rate and nature of sensitivity of physiological measures to emotional load is unknown (e.g., low complexity could trigger more electrodermal activity due to boredom and frustration; see also, Mandrick et al. 2016).

2. Addressing energetic interactions. The complex differential effects of emotional load on MWL in various contexts are by far unclear, so that practitioners do not know how to tackle them (cf., cognitive overload can lead to frustration, depleting MWL resources, in turn triggering coping behavior changing the cognitive appraisals of the situation, etc.). 
In the light of the intricate multidimensionality of MWL illustrated by our concept analysis, one could however argue that that no single definition and measurement procedure can cover the integral complexity of MWL (cf., Xie and Salvendy 2000), since ergonomics is all about context (Young and Stanton 2002). Our conceptual definition does not aspire to fully explain all MWL's nuances and sensitivities from an essentially cognitive psychological point of view. We extracted our definition from a concept analysis revealing the literature's common grounds and formulated it in a adequately generic way. The complexity then lies in comparing and cultivating the various contextualizations of this generic definition. In order to do so rigorously, we developed an explanatory framework of the dynamic processes affecting and following from MWL. Concretely, this framework allows for multifactorial contextualized operationalizations (as called for by de Waard and LewisEvans 2014 and Matthews et al. 2015), but most importantly, imbedded in a common, comparable structure. Such framework (when iterated upon) could then eventually help in developing measurement procedures covering (at least most of) the complexity of MWL.

To achieve such goals, we suggest that future research and practice could apply and build on our framework as follows: First, by providing a systematic explicitation of variables and measures for all empirical investigations. That is, addressing the choice of variables (e.g., because of replication motivations, practical urgency or theoretical unclarity), how exactly variables were manipulated (compared to other empirical accounts) and measured (e.g., which electrodermal components were significant and importantly, which were not), which aspects of MWL could have been triggered (e.g., mainly vigilance or working memory span), imperatively, the possible ways in which they could have been affected by and affect other variables and how they were (if any) controlled for, and finally, by designing a specific operational definition. By weighing various of such systematic accounts against each other, the specific sensitivities of measures to various cognitive work demands for different individuals in different contexts could be revealed in a systematic and comparable way (see also the suggestion raised by Matthews et al. 2015a). Next to this, results will be more generalizable and more free of confounding variance, eventually merging into stronger theorization (perhaps more at the representational than the operational side, see de Winter 2014) and a more robust set of contextualizable guidelines for practitioners.

Secondly, future work could try to maximize measurement triangulation. Physiological measures, subjective measures and performance measures all have their unique advantages and drawbacks (Paxion et al. 2014). When cross-verified through methodological triangulation (Denzin 1970), they can strongly add to data interpretation (cf., low subjective estimations of MWL, but high measured physiological arousal because of boredom and frustration) and measurement validation (cf., Matthews et al. 2015b; Matthews 2016). The multidimensional nature of MWL could hence be reflected systematically in its operationalizations.

Third, implementation of physical load and emotional load effects in measurement procedures and explanatory frameworks is highly desirable. Here, we did not expand on this anomaly present in the current stateof-science. Important questions therefore still remain unanswered. How do coping and other appraisals exactly relate to work-related emotional load, coping behavior and its energetic effects on MWL, for example. Although emotional load is no conditio sine qua non in MWL, in reality, adverse emotional experiences (such as performance anxiety) are sometimes hard to manage as a practitioner or researcher. The intention should however be to exclude them as much as possible from the work space. When measuring, subjective measures trying to unveil such complex cognitive estimations leading to emotions should therefore always be included in assessment procedures (cf., de Waard and Lewis-Evans 2014; Matthews 2016). Appraisal theory (cf., Scherer et al. 2001) could be of strong value to do so. Accelerometers and motion tracking sensors, for instance, and specific (pilot) experiments are then necessary to investigate potential confounding variance of physical load in (quasi) real-life mobile settings.

Fourth, building on the effects covered by cognitive load theory (Sweller 1988; 1994) and cognitive theory of multimedia learning (Mayer and Moreno 2003) could present more opportunities to manipulate and understand MWL's dynamics (in instruction design, for example), while also longer term learning effects will be addressed 
more profoundly. In all, we summarize these four suggestions for future work and practice in Table 3 and add a brief research example from one of our own research projects.

Table 3 Some suggestions for future research and practice

1. Systematic explicitation of all variables and how they (could) interact.

2. Maximization of measurement triangulation.

3. Implementation of emotional load and physical load in measurement procedures and interpretative models.

4. Building on the effects covered by cognitive load theory (Sweller 1988; 1994) and cognitive theory of multimedia learning (Mayer and Moreno 2003).

$\rightarrow$ Brief research example:

The current authors are finalizing a first empirical study based on the suggestions mentioned here. This respective study manipulated task complexity mainly through visual-spatial and working memory span properties of a manual assembly (based on, Richardson et al. 2004; Richardson et al. 2006), triggering mainly selective and divided attention related to visualspatial processing (instead of, e.g., arithmetic processing). Our hypotheses stated that pupil dilation and a subjective questionnaire would indicate the difference between two levels of complexity. The operational definition would state that the "mental workload will be reflected in a subjective estimation and in the pupillometry, revealing the interplay between participants' limited visual sensory modalities, etc., (cf., Wickens 2002, 2008), and the visual-spatial work demands and the working memory span demands (e.g., remembering the amount of fastening points) being exposed to". Moderating effects controlled for and represented in a contextualized framework are visual-spatial intelligence, dexterity, assembly experience, etc. Emotional load was controlled for through subjective measures and methodologically, while split-attention effect was controlled for methodologically only.

When operationalizing the presented definition, framework and suggestions in a first step, many interpretations will still be possible and fine-grained interactions between the multiplicity of variables will still need to be discovered. Such explanatory framework therefore requires intensive field-testing and widespread practical and theoretical iteration, possibly reconfiguring its premises and refining its explanatory structure. We hope that our findings could serve as one theory-driven leverage to do so and steer theoretical accumulation, measurement validation and practical implementation into an elucidated and more stipulated direction.

\section{Conclusion}

The present account first ran a concept analysis covering 82 theoretical and applied references related to MWL and revealed the concept's nebulous nature. Various proposed definitions were found to arbitrarily include and exclude defining variables, while also residing on different levels of abstraction. Mediators and moderators are only distantly touched upon, often conceptualized incorrectly and not included into an overarching explanatory framework. Emotional load variables then, are frequently operationalized in measurement, while no theoretical agreement is present concerning its explanatory and conceptual role. As a consequence, the concept of MWL found itself trapped in a complex knot, sustaining the confusion around its understanding and refraining effective theory building and applied optimization. The presented concept analysis (centralizing MWL's antecedents, defining attributes and consequences), definition, explanatory framework and suggestions for future research and practice could show to be a first step in disentangling the properties and processes of MWL and all its interrelated variables. The authors therefore look forward to learn from applications of and iterations on these notions, eventually sparking effective mental workload optimization initiatives in a wide array of contexts. 


\section{References}

Aasman J, Mulder G, Mulder LJM (1987) Operator effort and the measurement of heart-rate variability. Hum Factors 29:161-170. doi: 10.1177/001872088702900204

Andre AD (2001) The value of workload in the design and analysis of consumer products. In: Hancock PA, Desmond PA (eds) Stress, workload, and fatigue. L. Erlbaum., Mahwah, NJ, pp 373-382

Antonenko P, Paas F, Grabner R, van Gog T (2010) Using electroencephalography to measure cognitive load. Educ Psychol Rev 22:425-438. doi: 10.1007/s10648-010-9130-y

Ayaz H, Shewokis PA, Bunce S, et al (2012) Optical brain monitoring for operator training and mental workload assessment. Neuroimage 59:36-47. doi: 10.1016/j.neuroimage.2011.06.023

Backs RW, Lenneman JK, Wetzel JM, Green P (2003) Cardiac measures of driver workload during simulated driving with and without visual occlusion. Hum Factors J Hum Factors Ergon Soc 45:525-538. doi: 10.1518/hfes.45.4.525.27089

Baddeley A (1992) Working memory. Science 255:556-559. doi: 10.1126/science.1736359

Baddeley A (2000) The episodic buffer: a new component of working memory? Trends Cogn Sci 4:417-423.

Baron RM, Kenny DA (1986) The moderator-mediator variable distinction in social psychological research: conceptual, strategic, and statistical considerations. J Pers Soc Psychol 51:1173-1182. doi: 10.1037/00223514.51.6.1173

Basil MD (2012) Multiple resource theory. In: Seel NM (ed) Encyclopedia of the Sciences of Learning. Springer US, Boston, MA, pp 2384-2385

Block RA, Hancock PA, Zakay D (2016) Physical load affects duration judgments: a meta-analytic review. Acta Psychol (Amst) 165:43-47. doi: 10.1016/j.actpsy.2016.01.002

Bollen KA (2010) Structural equations with latent variables. Wiley, New York

Boucsein W (2012) Electrodermal activity, 2nd edn. Springer, New York

Brookhuis KA, de Waard D (2000) Assessment of drivers' workload: performance, subjective and physiological indices. In: Hancock PA, Desmond PA (eds) Stress, workload, and fatigue. L. Erlbaum, Mahwah, NJ, pp 321-333

Brünken R, Plass JL, Leutner D (2003) Direct measurement of cognitive load in multimedia learning. Educ Psychol 38:53-61. doi: 10.1207/S15326985EP3801_7

Bruya B (2010) Effortless attention. a new perspective in the cognitive sciene of attention and action. MIT Press. A Bradford book, Cambridge, MA

Cain B (2007) A review of the mental workload literature. In: Report No.: RTO-TR-HFM-121-Part-II. Defence Research and Development Canada Toronto Human System Integration Section, Ontario, WT, pp 1-34

Chen F, Zhou J, Wang Y, et al (2016) Robust multimodal cognitive load measurement. Springer, Switzerland

Choi H-H, van Merriënboer JJG, Paas F (2014) Effects of the Physical environment on cognitive load and learning: towards a new model of cognitive load. Educ Psychol Rev 26:225-244. doi: 10.1007/s10648014-9262-6

Crawford LE, Cacioppo JT (2002) Learning where to look for danger: integrating affective and spatial information. Psychol Sci 13:449-453. doi: 10.1111/1467-9280.00479

Csikszentmihalyi M (1975) Beyond boredom and anxiety, 1st edn. Jossey-Bass, San Francisco

de Waard D, Lewis-Evans B (2014) Self-report scales alone cannot capture mental workload. Cogn Technol Work 16:303-305. doi: 10.1007/s10111-014-0277-z

de Winter JCF (2014) Controversy in human factors constructs and the explosive use of the NASA-TLX: a measurement perspective. Cogn Technol Work 16:289-297. doi: 10.1007/s10111-014-0275-1

Denzin K (1970) The research act. Aldine, Chicago

Engström J, Bärgman J, Nilsson D, et al (2017) Great expectations: a predictive processing account of automobile driving. Theor Issues Ergon Sci 19:156-194. doi: 10.1080/1463922X.2017.1306148

Estes S (2015) The workload curve: subjective mental workload. Hum Factors 57:1174-1187. doi: $10.1177 / 0018720815592752$

Fairclough SH (2001) Mental effort regulation and the functional impairment of the driver. In: Hancock PA, Desmond PA (eds) Stress, workload, and fatigue. L. Erlbaum., Mahwah, NJ,

Gaillard AWK (1993) Comparing the concepts of mental load and stress. Ergonomics 36:991-1005. doi: $10.1080 / 00140139308967972$

Galvin EC, Wills T, Coffey A (2017) Readiness for hospital discharge: a concept analysis. J Adv Nurs 1-11. doi: 10.1111 /jan.13324

Gerjets P, Scheiter K, Cierniak G (2009) The scientific value of cognitive load theory: a research agenda based on the structuralist view of theories. Educ Psychol Rev 21:43-54. doi: 10.1007/s10648-008-9096-1

Gopher D, Donchin E (1986) Workload: an examination of the concept. In: Boff KR, Kaufman L, Thomas JP (eds) Handbook of perception and human performance. Vol. 2. Cognitive processes and performance. John Wiley \& Sons, Oxford, England, pp 1-49 
Grassmann M, Vlemincx E, von Leupoldt A, Van den Bergh O (2017) Individual differences in cardiorespiratory measures of mental workload: an investigation of negative affectivity and cognitive avoidant coping in pilot candidates. Appl Ergon 59:274-282. doi: 10.1016/j.apergo.2016.09.006

Guastello SJ, Shircel A, Malon M, Timm P (2014) Individual differences in the experience of workload. Theor Issues Ergon Sci 16:20-52.

Hancock PA (1996) Effects of control order, augmented feedback, input device and practice on tracking performance and perceived workload. Ergonomics 39:1146-1162.

Hancock PA, Warm JS (1989) A dynamic model of stress and sustained attention. Hum Factors J Hum Factors Ergon Soc 31:519-537. doi: 10.1177/001872088903100503

Hart SG, Staveland LE (1988) Development of NASA-TLX (Task Load Index): results of empirical and theoretical research. In: Hancock PA, Meshkati N (eds) Human mental workload. Elsevier Science Publishers B.V. (North-Hollland), Amsterdam, pp 139-183

Hart SG, Wickens CD (2010) Cognitive workload. In: NASA Human Integration Design Handbook (HIDH). NASA, Washington, D.C., pp 190-222

Hilburn B, Jorna PGAM (2001) Stress, workload, and fatigue. In: Hancock PA, Desmond PA (eds) Stress, workload, and fatigue. L. Erlbaum, Mahwah, NJ,

Hockey GRJ (1997) Compensatory control in the regulation of human performance under stress and high workload: a cognitive energetical framework. Biol Psychol 45:73-93. doi: 10.1016/S0301-0511(96)052234

Jipp M, Ackerman PL (2016) The impact of higher levels of automation on performance and situation awareness. J Cogn Eng Decis Mak 10:138-166. doi: 10.1177/1555343416637517

Johannsen G (1979) Workload and workload measurement. In: Moray N (ed) Mental Workload: its theory and measurement. Plenum Press, New York, pp 3-11

Kahneman D (1973) Attention and effort. Am J Psychol 88:339. doi: 10.2307/1421603

Kramer AF, Sirevaag EJ, Braune R (1987) A psychophysiological assessment of operator workload during simulated flight missions. Hum Factors J Hum Factors Ergon Soc 29:145-160. doi: $10.1177 / 001872088702900203$

Langner R, Eickhoff SB (2013) Sustaining attention to simple tasks: a meta-analytic review of the neural mechanisms of vigilant attention. Psychol Bull 139:870-900. doi: 10.1037/a0030694

Layer JK, Karwowski W, Furr A (2009) The effect of cognitive demands and perceived quality of work life on human performance in manufacturing environments. Int J Ind Ergon 39:413-421. doi: 10.1016/j.ergon.2008.10.015

Longo L (2015) A defeasible reasoning framework for human mental workload representation and assessment. Behav Inf Technol 34:758-786. doi: 10.1080/0144929X.2015.1015166

Mandler G (1979) Thought processes, consciousness, and stress. In: Hamilton V, Warburton DM (eds) Human stress and cognition: an information processing approach. John Wiley \& Sons, Inc., New York, pp 179201

Mandrick K, Chua Z, Causse M, et al (2016) Why a comprehensive understanding of mental workload through the measurement of neurovascular coupling is a key issue for neuroergonomics? Front Hum Neurosci 10:1-5. doi: 10.3389/fnhum.2016.00250

Matthews G (2016) Multidimensional profiling of task stress states for human factors: a brief review. Hum Factors J Hum Factors Ergon Soc 58:801-813. doi: 10.1177/0018720816653688

Matthews G, Campbell SE (2010) Dynamic relationships between stress states and working memory. Cogn Emot 24:357-373. doi: 10.1080/02699930903378719

Matthews G, Campbell SE, Falconer S, et al (2002) Fundamental dimensions of subjective state in performance settings: task engagement, distress, and worry. Emotion 2:315-340. doi: 10.1037/1528-3542.2.4.315

Matthews G, Reinerman-Jones L, Wohleber R, et al (2015a) Workload is multidimensional, not unitary: what now? In: Schmorrow DD, Fidopiastis CM (eds) Foundations of uugmented cognition: 9th International Conference, AC 2015, held as Part of HCI International 2015, Los Angeles, CA, USA, August 2-7, 2015, Proceedings. Springer International Publishing, Cham, pp 44-55

Matthews G, Reinerman-Jones LE, Barber DJ, Abich J (2015b) The psychometrics of mental workload: Multiple measures are sensitive but divergent. Hum Factors 57:125-143. doi: 10.1177/0018720814539505

Matthews G, Szalma J, Rose A, et al (2013) Profiling task stress with the dundee stress state questionnaire. In: Cavalcanti L, Azevedo S (eds) Psychology of stress: new research. Nova Science Publishers, Hauppage, NY, pp 49-91

Mautone PD, Mayer RE (2001) Signaling as a cognitive guide in multimedia learning. J Educ Psychol 93:377389. doi: 10.1037/0022-0663.93.2.377

Mayer R, Moreno R (2003) Nine ways to reduce cognitive load in multimedia learning. Educ Psychol 38:43-52.

Mayer RE, Sims VK (1994) For whom is a picture worth a thousand words? Extensions of a dual-coding theory of multimedia learning. J Educ Psychol 86:389-401. doi: 10.1037/0022-0663.86.3.389 
McGrath JE (1976) Stress and behavior in organizations. In: Dunnette MD (ed) Handbook of industrial and organizational psychology. Rand McNally, Chicago, pp 1351-1395

Mehta RK, Parasuraman R (2013) Neuroergonomics: a review of applications to physical and cognitive work. Front Hum Neurosci. doi: 10.3389/fnhum.2013.00889

Moray N (1979) Mental workload: its theory and measurement. Plenum, New York

Neerincx MA, van Doorne H, Ruijsendaal M (2000) Attuning computer-supported work to human knowledge and processing capacities in ship control centres. In: Schraagen JMC, Chipman SE, Shalin VL (eds) Cognitive task analysis. Erlbaum, Mahwah, NJ,

Norman DA, Bobrow DG (1975) On data-limited and resource-limited processes. Cogn Psychol 7:44-64.

O’Donnell RD, Eggemeier FT (1986) Workload assessment methodology. In: Boff KR, Kaufman L, Thomas JP (eds) Handbook of perception and human performance. Volume 2. Cognitive processes and performance. Wiley Interscience, New York, p 42/1-42/9

Oviatt S (2006) Human-centered design meets cognitive load theory: designing interfaces that help people think. In: ACM International Conference on Multimedia. ACM, Santa Barbara, CA, USA, pp 871-880

Paas FGWC, Tuovinen J, Tabbers H, van Gerven PWM (2003) Cognitive load measurement as a means to advance cognitive load theory. Educ Psychol 38:63-71.

Parasuraman R, Hancock PA (2001) Adaptive control of mental workload. In: Hancock PA, Desmond PA (eds) Stress, workload and fatigue. L. Erlbaum, Mahwah, NJ, pp 305-320

Parasuraman R, Wilson GF (2008) Putting the brain to work: neuroergonomics past, present, and future. Hum Factors 50:468-474. doi: 10.1518/001872008X288349

Paxion J, Galy E, Berthelon C (2014) Mental workload and driving. Front Psychol 5:1-11. doi: 10.3389/fpsyg.2014.01344

Pickup L, Wilson JR, Sharpies S, et al (2005) Fundamental examination of mental workload in the rail industry. Theor Issues Ergon Sci 6:463-482. doi: 10.1080/14639220500078021

Pillay HK (1997) Cognitive load and assembly tasks: effect of instructional formats on learning assembly procedures. Educ Psychol 17:285-299. doi: 10.1080/0144341970170304

Proctor RW, Vu K-PL (2012) Selection and control of action. In: Salvendy G (ed) Handbook of human factors and ergonomics, 4th edn. John Wiley \& Sons, Inc., Hoboken, NJ, pp 95-116

Reid GB, Nygren TE (1988) The subjective workload assessment technique: a scaling procedure for measuring mental workload. In: Hancock A, Meshkati N (eds) Human mental workload. Elsevier Science Publishers B.V., Amsterdam: North-Holland, pp 185-218

Richardson M, Jones G, Torrance M (2004) Identifying the task variables that influence perceived object assembly complexity. Ergonomics 47:945-964. doi: 10.1080/00140130410001686339

Richardson M, Jones G, Torrance M, Baguley T (2006) Identifying the Task Variables That Predict Object Assembly Difficulty. Hum Factors J Hum Factors Ergon Soc 48:511-525. doi: $10.1518 / 001872006778606868$

Ricker TJ, Vergauwe E, Hinrichs GA, et al (2014) No recovery of memory when cognitive load is decreased. J Exp Psychol Learn Mem Cogn Cogn 1-9. doi: http://dx.doi.org/10.1037/xlm0000084

Rubio S, Díaz E, Martín J, Puente JM (2004) Evaluation of subjective mental workload: a comparison of SWAT, NASA-TLX, and Workload Profile methods. Appl Psychol 53:61-86. doi: 10.1111/j.14640597.2004.00161.x

Salvendy G (2012) Handbook of human factors and ergonomics. John Wiley \& Sons, Inc., Hoboken, NJ

Scherer KR, Shorr A, Johnstone T (2001) Appraisal processes in emotion: theory, methods, research. Oxford University Press., Canary, NC

Schneider W, Shiffrin RM (1977) Controlled and automatic human information processing: I. Detection, search, and attention. Psychol Rev 84:1-66. doi: 10.1037/0033-295X.84.1.1

Shakouri M, Ikuma LH, Aghazadeh F, Nahmens I (2018) Analysis of the sensitivity of heart rate variability and subjective workload measures in a driving simulator: the case of highway work zones. Int J Ind Ergon 66:136-145. doi: 10.1016/j.ergon.2018.02.015

Shaw FA, Park SJ, Bae J, et al (2018) Effects of roadside distractors on performance of drivers with and without attention deficit tendencies. Transp Res Part F Traffic Psychol Behav. doi: 10.1016/j.trf.2018.02.013

Sörqvist P, Dahlström Ö, Karlsson T, Rönnberg J (2016) Concentration: the neural underpinnings of how cognitive load shields against distraction. Front Hum Neurosci. doi: 10.3389/fnhum.2016.00221

Staal MA (2004) Stress, cognition, and human performance: a literature review and conceptual framework. NASA Tech Memo 212824 pp 1-170.

Stevenson A (2010) Oxford dictionary of English, 3rd edn. Oxford University Press, Oxford, UK

Stork S, Schubö A (2010) Human cognition in manual assembly: theories and applications. Adv Eng Informatics 24:320-328. doi: 10.1016/j.aei.2010.05.010

Stramler JH (1992) The dictionary for human factors/ergonomics. CRC Press, Boca Raton, FL

Sweller J (1988) Cognitive load during problem solving: effects on learning. Cogn Sci 12:257-285. doi: 


\section{$10.1016 / 0364-0213(88) 90023-7$}

Sweller J (1994) Cognitive load theory, learning difficulty, and instructional design. Learn Instr 4:295-312. doi: 10.1016/0959-4752(94)90003-5

Tindall-Ford S, Chandler P, Sweller J (1997) When two sensory modes are better than one. J Exp Psychol Appl 3:257-287. doi: 10.1037/1076-898X.3.4.257

Van de Ven B, Vlerick P, de Jonge J (2008) The interplay of job demands, job resources and cognitive outcomes in informatics. Stress Heal 24:375-382. doi: 10.1002/smi.1192

Venkatesh V, Morris MG, Davis FD, Davis GB (2003) User accceptance of information technology: toward a unified view. MIS Q 27:425-478. doi: 10.2307/30036540

Vidulich MA, Tsang PS (1986) Techniques of subjective workload assessment: a comparison of SWAT and the NASA-Bipolar methods. Ergonomics 29:1385-1398. doi: 10.1080/00140138608967253

Wacker JG (2004) A theory of formal conceptual definitions: developing theory-building measurement instruments. J Oper Manag 22:629-650. doi: 10.1016/j.jom.2004.08.002

Walker LO, Avant KC (2011) Strategies for theory construction in nursing, 5th edn. Prentice Hall, Upper Saddle River, NJ

Warm JS, Parasuraman R, Matthews G (2008) Vigilance requires hard mental work and is stressful. Hum Factors J Hum Factors Ergon Soc 50:433-441. doi: 10.1518/001872008X312152

Wickens CD (2002) Multiple resources and performance prediction. Hum Factors J Hum Factors Ergon Soc 3:159-177.

Wickens CD (2008) Multiple resources and mental workload. Hum Factors J Hum Factors Ergon Soc 50:449455. doi: 10.1518/001872008X288394.

Wickens CD (2017) Mental workload: assessment, prediction and consequences. In: Longo L, Leva MC (eds) Human mental workload: models and applications: First International Symposium, H-WORKLOAD 2017, Dublin, Ireland, June 28-30, 2017, revised selected papers. Springer International Publishing, Cham, pp $18-29$

Wickens CD, Carswell CM (2012) Chapter 5. Information processing. In: Salvendy G (ed) Handbook of human factors and ergonomics, 4th edn. John Wiley \& Sons, Inc., Hoboken, NJ, pp 117-161

Wickens CD, Hollands JG (2000) Engineering psychology and human performance, 3rd edn. Prentice-Hall, Upper Saddle River, NJ

Wickens CD, Tsang P (2014) Handbook of human-systems integration. In: F. Durso (ed) Handbook of humansystems integration. American Psychological Association, Washington, DC,

Xie B, Salvendy G (2000) Prediction of mental workload in single and multiple tasks environments. Int J Cogn Ergon 4:213-242. doi: 10.1207/S15327566IJCE0403_3

Young MS, Brookhuis KA, Wickens CD, Hancock PA (2014) State of science: mental workload in ergonomics. Ergonomics 139:1-17. doi: 10.1080/00140139.2014.956151

Young MS, Stanton NA (2001) Mental workload: theory, measurement and application. In: Karwowski W (ed) International encyclopedia of ergonomics and human factors. Taylor \& Francis, London, pp 507-509

Young MS, Stanton NA (2002) It's all relative: defining mental workload in the light of Annett's paper. Ergonomics 45:1018-1020.

Zijlstra FRH (1993) Efficiency in work behaviour: a design approach for modern tools. Dissertation, Delft University Press, Delft 